\title{
Serum/Synovial Fluid Urate Ratio as an Indicator for Distinguishing Gouty Arthritis From Other Arthritides
}

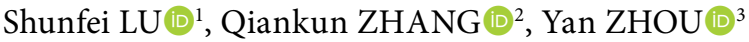 \\ ${ }^{1}$ Lishui University, College of Medicine and Health, Lishui, China \\ ${ }^{2}$ Lishui Central Hospital, Rheumatology, Lishui, China \\ ${ }^{3}$ The First Affiliated Hospital of Wenzhou Medical University, Rheumatology, Wenzhou, China
}

\begin{abstract}
Objectives: This study aims to compare the serum/synovial fluid (SF) urate ratio of gouty arthritis and other arthritides and investigate whether this ratio may be an indicator for distinguishing gouty arthritis from other arthritides.

Patients and methods: Paired serum and SF samples from 70 patients (38 males, 32 females; mean age 57.9 years; range, 27.5 to 78.4 years) comprised of 20 patients with gout, 20 patients with rheumatoid arthritis and 30 patients with osteoarthritis were collected simultaneously for urate measurement. Patient data were recorded including demographic data (age, sex), body mass index, estimated glomerular filtration rate, comorbidities (diabetes, hypertension and dyslipidemia), disease duration (from the first symptoms), pain duration before arthrocentesis at this time, presence of tophi or not, serum albumin, erythrocyte sedimentation rate, C-reactive protein and SF white blood cell count.

Results: Gout patients had highest levels of urate in both serum and SF among the three groups $(p<0.001)$. The serum/SF urate ratio of gout patients was significantly lower among the three groups $(p<0.001)$. Receiver-operating characteristic curve analysis demonstrated that serum/SF urate ratio can predict the extent of gouty arthritis (the value of area under the curve was $0.867, p<0.001$ ).

Conclusion: Serum/SF urate ratio may be an indicator for distinguishing gouty arthritis from other arthritides.

Keywords: Gout; serum, synovial fluid, urate.
\end{abstract}

The detection of monosodium urate (MSU) crystals in the synovial fluid (SF) by compensated polarized light microscopy was recognized as a gold standard for the diagnosis of gouty arthritis., ${ }^{1,2}$ However, in previous studies, MSU crystals were difficult to be found in 5-48\% of gouty effusions, particularly in cases with a low SF leukocyte count. $^{3-7}$ The failure to detect crystals in the SF may be mainly due to observer error and low load of MSU crystals. ${ }^{8,9}$

The synovial membrane is a semi-permeable membrane, which permits urate pass freely through into the SF, so the levels of urate between the plasma and SF wound be similar. ${ }^{10}$ However, this balance may be changed in patients with gout. The serum urate might drop to normal levels during gouty attacks, ${ }^{11}$ and at the same time, the MSU crystals would be lysed by neutrophil peroxidases or by the reduction of the SF $\mathrm{pH} .{ }^{12,13}$ Since decreased serum urate and increased SF urate resulted from a digestion of the MSU crystals in SF, we hypothesized that the serum/SF urate ratio would be lower in gouty arthritis than other arthritides. In this study, we aimed to compare the serum/SF urate ratio of gouty arthritis and other arthritides and investigate whether this ratio may be an indicator for distinguishing gouty arthritis from other arthritides.

Received: October 22, 2017 Accepted: February 28, 2018 Published online: May 20, 2018

Correspondence: Yan Zhou, MD. The First Affiliated Hospital of Wenzhou Medical University, Rheumatology, 325000 Wenzhou, China. Tel: 86-578-2681234 e-mail: 215692141@qq.comr 


\section{PATIENTS AND METHODS}

Patients were enrolled between February 2013 and February 2016 at Lishui Central Hospital. Of 101 patients screened, 70 eligible patients (38 males, 32 females; mean age 57.9 years; range, 27.5 to 78.4 years) were enrolled, consisting of 20 patients with gout, 20 patients with RA and 30 patients with osteoarthritis (OA). Inclusion criteria were: (i) Gouty arthritis, rheumatoid arthritis (RA) and OA involving the knee and knee swelling with effusions by examination. (ii) Gouty arthritis was defined as presence of MSU in SF determined by compensated polarized light microscopy previously or currently. (iii) RA and OA were both in accordance with their respective diagnostic criteria. ${ }^{14,15}$ Exclusion criteria were: (i) Patients developing more than one joint disease. (ii) The use of warfarin or antiplatelet therapy. (iii) The presence of infection. The study protocol was approved by the Lishui Central Hospital Ethics Committee. A written informed consent was obtained from each patient. The study was conducted in accordance with the principles of the Declaration of Helsinki.

The serum and SF were collected simultaneously, with $3 \mathrm{~mL}$ serum and at least $3 \mathrm{~mL}$ SF for urate measurement. All SF samples were searched for MSU crystals by compensated polarized microscopy. The urate of both serum and SF was determined by uricase method using automated analyze. Both blood and SF samples were analyzed within three hours after collections.

The following data were also recorded: demographics (age, sex), body mass index, estimated glomerular filtration rate, comorbidities (diabetes, hypertension and dyslipidemia), disease duration (from the first symptoms), pain duration before arthrocentesis at this time, presence of tophi or not, serum albumin, erythrocyte sedimentation rate (ESR), C-reactive protein (CRP) and SF white blood cell count.

\section{Statistical analysis}

Statistical analysis was conducted by using SPSS version 16.0 software (SPSS Inc., Chicago, IL, USA). Data were expressed as percentage, mean (standard deviation), or median interquartile range, where appropriate. One-way analysis of variance or Chi square $\left(\chi^{2}\right)$ test was used to compare differences among the groups. Pearson's

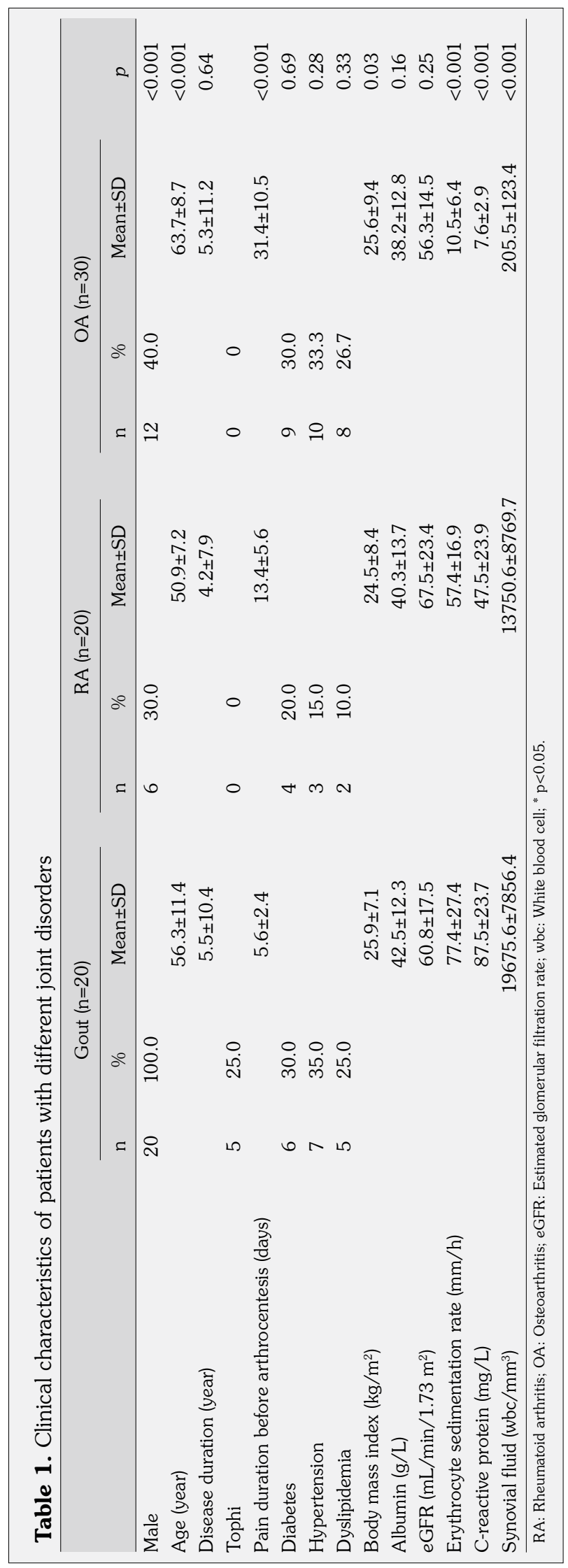


Table 2. Serum and synovial fluid levels of urate of patients with different joint disorders

\begin{tabular}{|c|c|c|c|c|}
\hline & Gout $(n=20)$ & $\mathrm{RA}(\mathrm{n}=20)$ & $\mathrm{OA}(\mathrm{n}=30)$ & \\
\hline Variable & Mean \pm SD & Mean \pm SD & Mean \pm SD & $p$ \\
\hline Serum urate $(\mathrm{mg} / \mathrm{dL})$ & $7.9 \pm 1.2$ & $6.4 \pm 0.7$ & $6.6 \pm 1.0$ & $<0.001$ \\
\hline SF urate $(\mathrm{mg} / \mathrm{dL})$ & $8.2 \pm 1.1$ & $6.1 \pm 0.7$ & $6.4 \pm 0.7$ & $<0.001$ \\
\hline Serum/SF urate ratio & $1.0 \pm 0.0$ & $1.1 \pm 0.0$ & $1.0 \pm 0.0$ & $<0.001$ \\
\hline
\end{tabular}

correlation test was used to assess the association between the urate level of serum and SF in both groups. Additionally, a receiver operating characteristics (ROC) curve was plotted for serum/SF ratio of urate with gouty arthritis and the like-hood ratio was also calculated. A $p$ value $<0.05$ was regarded as statistically significant.

\section{RESULTS}

The 70 SF samples were all obtained from knee joints. Among the 20 patients of gout, 15 patients were in attacks of acute gout and five patients in inter-critical period; three patients were not found to have MSU crystals in SF at this time (all in the inter-critical period), but they all had previousMSU-crystal-proven-gout. Clinical characteristics of the study population were shown in Table 1. Obviously, the gout patients had shortest duration before arthrocentesis, had highest levels of ESR, CRP and white blood cells count of SF among the three groups $(p<0.001)$. As shown in Table 2 , the gout patients had highest urate levels in both

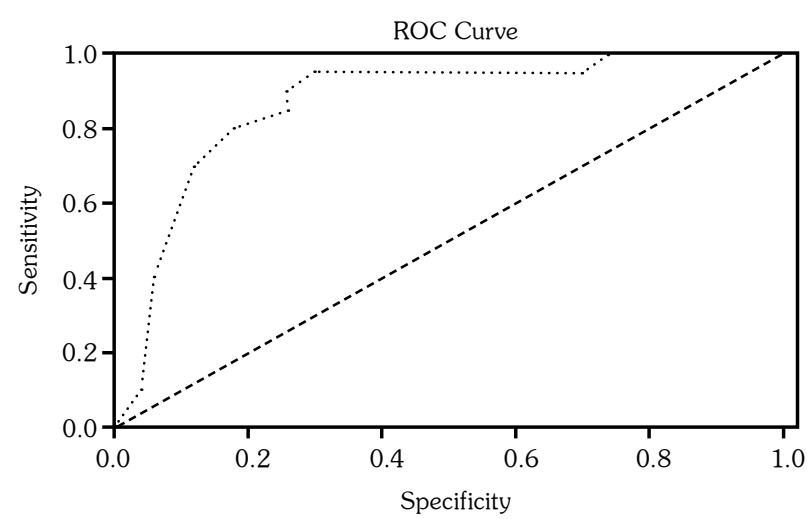

Figure 1. Receiver-operating characteristic (ROC) curve analysis showing prognostic value of serum/synovial fluid urate ratio in predicting gouty arthritis. serum and SF among the three groups $(p<0.001)$. Interestingly, we also found strong correlations of urate levels between paired samples of serum and $\mathrm{SF}$ in gout, RA and OA groups $(\mathrm{r}=0.82, \mathrm{p}<0.001$; $r=0.86, p<0.001 ; r=0.90, p<0.001$, respectively). A comparison of the serum/SF urate ratio among the three groups showed that the serum/SF urate ratio of gout patients were significantly lower $(p<0.001)$.

Receiver operating characteristic curve analysis (Figure 1) showed that serum/SF urate ratio can predict the extent of gouty arthritis (area under the curve value was $0.867, \mathrm{p}<0.001$, and the $95 \%$ confidence interval was $0.775-0.959)$. The optimal serum/SF urate ratio cut-off value for predicting gouty arthritis was 1.005 . This cut-off value had a sufficient sensitivity and specificity (sensitivity $=95.0 \%$, specificity $=70.0 \%$ ) and the likelihood ratio value calculated for the optimal cut-off point was 3.16 .

\section{DISCUSSION}

In recent years, the diagnostic criteria of gout have been constantly updated and improved. The 2015 Gout classification criteria from American College of Rheumatology/European League Against Rheumatism collaborative had included ultrasound and double-energy computed tomography examination, which was thought to be a great progress. ${ }^{16}$ However, although there were many criteria for the diagnosis of gout, none of the criteria had both high sensitivity and specificity. ${ }^{17}$ As the researches about SF urate increase, we believe that the SF urate will play an important role in the diagnosis of gout in the future.

The present study showed that serum urate level positively correlated with SF urate level in all groups. This was consistent with some 
previous studies. Gudbjörnsson et al. ${ }^{18}$ found that serum urate level strongly correlated with SF urate level $(r=0.76, p<0.01)$ in inflammatory arthritis patients. Wangkaew et al. ${ }^{19}$ reported a strongly positive correlation $(\mathrm{r}=0.81-0.91, \mathrm{p}<0.01)$ between serum urate level and SF urate level in both inflammatory and non-inflammatory arthritis patients. The reason might be that the synovial membrane allowed urate and other small molecules to pass freely through into the SF, which could be considered as a dialysate of blood plasma. ${ }^{10,20}$ However, Beutler et al. ${ }^{21}$ found that SF urate level correlated with serum level in patients with gout and non-inflammatory arthritis but not with RA. Such differences might have resulted from the fact that the synovial membrane of RA was less permeable to uric acid than other joint disorders. ${ }^{22}$

Uric acid was a weak acid that circulated as the urate anion under physiologic conditions. ${ }^{23}$ The general process of MSU crystallization was thought to depend on both hyperuricemia and other factors, including cold temperature and acidic environment. ${ }^{24}$ On the other hand, the heat produced by the inflammatory gouty arthritis may result in the subsequent dissolution of crystals. Theoretically, the elevations of SF urate levels seen in gout could be unique for this disease and most likely reflect the crystal dissolution in joints.

This study also demonstrated that the serum/SF urate ratio of gout patients was significantly lower than the other two groups. This was similar to some previous studies. Rozin et al. ${ }^{25}$ had studied six gout patients with normal serum urate and demonstrated that the SF urate level was higher than serum in these patients. Tiliakos and Tiliakos ${ }^{26}$ showed that the SF urate level during attacks of acute gout was higher than serum and gradually equalized to the serum when the arthritis relieved. Wangkaew et al. ${ }^{19}$ found that the serum/SF ratio of urate of gout patients was lower than that in patients with calcium pyrophosphate dihydrate deposition disease, RA, septic arthritis, ankylosing spondylitis and OA, although there was no significant difference.

There are many methods to improve the crystal detection rate including repeated aspiration from different involved joints or the same joint over period of hours to months, analyst training and cytospin preparations. ${ }^{27,28}$ However, even so, there would be no crystals found in SF at some time, when the serum/SF urate ratio maybe a good tool to exclude the diagnosis of gout. We performed a ROC curve analysis and showed that serum/SF urate ratio can predict the extent of gouty arthritis. Also, the sum of sensitivity and specificity for the prediction of the extent of gouty arthritis was maximal at a serum/SF ratio of urate level of $\leq 1.005$.

There were two major limitations to our study. First, the present study had small sample sizes in all groups. Second, in the gouty arthritis group, 15 patients were in attacks of acute gout and five patients in inter-critical period; thus, these may have influenced both the serum and SF urate levels. Hence, further studies should compare larger groups of different arthritic diseases and distinguish the gout patients in the acute or inter-critical period.

In conclusion, our study demonstrated strongly positive correlations of urate levels between paired samples of serum and SF in all groups. The serum/SF urate ratio of gout patients was significantly lower than the non-gouty arthritis groups. In light of our observations, we suggest that serum/SF urate ratio $\leq 1.005$ may distinguish gouty arthritis from other arthritides.

\section{Declaration of conflicting interests}

The authors declared no conflicts of interest with respect to the authorship and/or publication of this article.

\section{Funding}

The authors received no financial support for the research and/or authorship of this article.

\section{REFERENCES}

1. Zhang W, Doherty M, Pascual E, Bardin T, Barskova V, Conaghan $P$, et al. EULAR evidence based recommendations for gout. Part I: Diagnosis. Report of a task force of the Standing Committee for International Clinical Studies Including Therapeutics (ESCISIT). Ann Rheum Dis 2006;65:1301-11.

2. Bardin T, Richette P. Definition of hyperuricemia and gouty conditions. Curr Opin Rheumatol 2014;26:186-91.

3. Pascual E, Sivera F, Andrés M. Synovial fluid analysis for crystals. Curr Opin Rheumatol 2011;23:161-9. 
4. Romanoff NR, Canoso JJ, Rubinow A, Spark EC. Gout without crystals on initial synovial fluid analysis. Postgrad Med J 1978;54:95-7.

5. Pascual E, Batlle-Gualda E, Martínez A, Rosas J, Vela P. Synovial fluid analysis for diagnosis of intercritical gout. Ann Intern Med 1999;131:756-9.

6. Bomalaski JS, Lluberas G, Schumacher HR Jr. Monosodium urate crystals in the knee joints of patients with asymptomatic nontophaceous gout. Arthritis Rheum 1986;29:1480-4.

7. Pascual E. Persistence of monosodium urate crystals and low-grade inflammation in the synovial fluid of patients with untreated gout. Arthritis Rheum 1991;34:141-5.

8. Schumacher HR, Jimenez SA, Gibson T, Pascual E, Traycoff R, Dorwart BB, et al. Acute gouty arthritis without urate crystals identified on initial examination of synovial fluid: Report of nine patients. Arthritis \& Rheumatology 1975;18:603-12.

9. Lumbreras B, Pascual E, Frasquet J, González-Salinas J, Rodríguez E, Hernández-Aguado I. Analysis for crystals in synovial fluid: training of the analysts results in high consistency. Ann Rheum Dis 2005;64:612-5.

10. Simkin PA. Synovial perfusion and synovial fluid solutes. Ann Rheum Dis 1995;54:424-8.

11. Badulescu M, Macovei L, Rezuş E. Acute gout attack with normal serum uric acid levels. Rev Med Chir Soc Med Nat Iasi 2014;118:942-5.

12. Pascual E, Addadi L, Andrés M, Sivera F. Mechanisms of crystal formation in gout-a structural approach. Nat Rev Rheumatol 2015;11:725-30.

13. Lam Erwin C-Y, Nancollas GH. The crystallization and dissolution of sodium. urate. $\mathrm{J}$ Cryst Growth 1981;53:215-23.

14. Altman R, Asch E, Bloch D, Bole G, Borenstein $\mathrm{D}$, Brandt $\mathrm{K}$, et al. Development of criteria for the classification and reporting of osteoarthritis. Classification of osteoarthritis of the knee. Diagnostic and Therapeutic Criteria Committee of the American Rheumatism Association. Arthritis Rheum 1986;29:1039-49.

15. Arnett FC, Edworthy SM, Bloch DA, McShane DJ, Fries JF, Cooper NS, et al. The American Rheumatism Association 1987 revised criteria for the classification of rheumatoid arthritis. Arthritis Rheum 1988;31:315-24.
16. Neogi T, Jansen TL, Dalbeth N, Fransen J, Schumacher HR, Berendsen D, et al. 2015 Gout classification criteria: an American College of Rheumatology/ European League Against Rheumatism collaborative initiative. Ann Rheum Dis 2015;74:1789-98.

17. Taylor WJ, Fransen J, Dalbeth N, Neogi T, Schumacher HR, Brown M, et al. Performance of classification criteria for gout in early and established disease. Ann Rheum Dis 2016;75:178-82.

18. Gudbjörnsson B, Zak A, Niklasson F, Hällgren R. Hypoxanthine, xanthine, and urate in synovial fluid from patients with inflammatory arthritides. Ann Rheum Dis 1991;50:669-72.

19. Wangkaew S, Kasitanon N, Hongsongkiat S, Tanasombat C, Sukittawut W, Louthrenoo W. A comparative study of serum and synovial fluid levels of uric acid between patients with gout and other arthritides. J Med Assoc Thai 2014;97:679-85.

20. Simkin PA, Pizzorno JE. Transynovial exchange of small molecules in normal human subjects. J Appl Physiol 1974;36:581-7.

21. Beutler AM, Keenan GF, Soloway S, Norden D, Luchi M, Schumacher HR Jr. Soluble urate in sera and synovial fluids from patients with different joint disorders. Clin Exp Rheumatol 1996;14:249-54.

22. Simkin PA. Synovial permeability in rheumatoid arthritis. Arthritis Rheum 1979;22:689-96.

23. Schlesinger N. Diagnosis of gout: clinical, laboratory, and radiologic findings. Am $\mathrm{J}$ Manag Care 2005;11:443-50.

24. Martillo MA, Nazzal L, Crittenden DB. The crystallization of monosodium urate. Curr Rheumatol Rep 2014;16:400.

25. Rozin AP, Braun-Moscovici Y, Balbir-Gurman A. Serum-synovial gradient data of normouricemic patients with history of gout and acute knee effusion. Clin Rheumatol 2006;25:886-8.

26. Tiliakos AN, Tiliakos NA. Total joint fluid urate in gout. J Clin Rheumatol 2004;10:250-1.

27. Robier C, Stettin M, Quehenberger F, Neubauer M. Cytospin preparations are superior to common smears in the detection of monosodium urate crystals in low-cellular synovial fluids. Clin Rheumatol 2014;33:1797-800.

28. Mader R. Repeated synovial fluid analysis may be needed to find crystals in gout. Clin Rheumatol 1998;17:549-50. 